\title{
Mo-derived perivascular macrophage recruitment protects against endothelial cell death in retinal vein occlusion
}

\author{
Christophe Roubeix ${ }^{1 * \dagger}$, Elisa Dominguez ${ }^{1 \dagger}$, William Raoul ${ }^{2}$, Xavier Guillonneau $^{1}$, Michel Paques ${ }^{1,3}$, \\ José-Alain Sahel ${ }^{1,3}$ and Florian Sennlaub ${ }^{1,4}$
}

\begin{abstract}
Background: To decipher the role of monocyte-derived macrophages (M $\varphi s)$ in vascular remodeling of the occluded vein following experimental branch retinal vein occlusion (BRVO).

Methods: The inflammation induced by laser-induced BRVO on mice retina was evaluated at different time points by RT-PCR looking at inflammatory markers mRNA level expression, Icam-1, Cd11 b, F4/80, CCl2, and Ccr2 and by quantification of Iba1-positive macrophage $(\mathrm{M} \varphi)$ density on Iba1-stained retinal flatmount. Repeated intraperitoneal EdU injection combined with liposome clodronate-induced monocyte (Mo) depletion in wildtype mice was used to

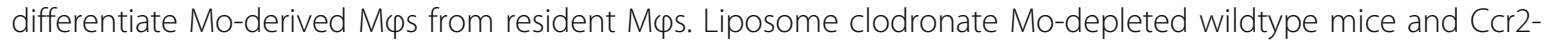
deficient mice were used to evaluate the role of all CCR2 ${ }^{+}$and $C C R 2^{\text {neg }}$ Mo-derived M $\varphi s$ on EC apoptosis in the occluded vein.

Results: cd11b, ICAM-1, F4/80, CCI2, and Ccr2 mRNA expression were increased 1, 3, and 7 days after vein occlusion. The number of parenchymal (parM $\varphi s$ ) and perivascular (vasM $M s$ ) macrophages was increased 3 and 7 days after BRVO. The systemic depletion of all circulating Mos decreased significantly the BRVO-induced parM $\varphi$ s and vasM $\varphi s$ macrophage accumulation, while the deletion of $C C R 2^{+}$-inflammatory Mo only diminished the accumulation of parM $\varphi s$, but not vasM $\varphi s$. Finally, apoptotic ECs of the vein were more numerous in fully depleted, liposome clodronate-treated mice, than in $\mathrm{Ccr}^{-/-}$mice that only lack the recruitment of $\mathrm{CCR}^{+}$inflammatory Mos.

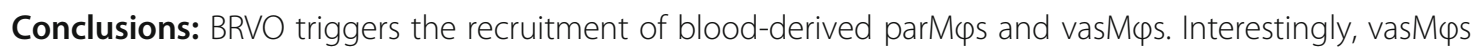
accumulation was independent of CCR2. The observation that the inhibition of the recruitment of all infiltrating M $\varphi$ s increases the vein EC apoptosis, while CCR2 deficiency does not, demonstrates that CCR2 ${ }^{\text {neg }}$ Mo-derived vasM $\varphi$ s protect the ECs against apoptosis in the occluded vein.
\end{abstract}

Keywords: BRVO, Perivascular macrophages, CCR2, Endothelial cells

\section{Introduction}

Branch retinal vein occlusion (BRVO) leads to extensive vascular remodeling and is an important cause of visual impairment due to vascular leakage and retinal edema. We have previously shown that experimental BRVO is associated with an acute wave of endothelial cell (EC) apoptosis of the occluded vein and the upstream

\footnotetext{
* Correspondence: chris.roubeix@gmail.com

${ }^{+}$Christophe Roubeix and Elisa Dominguez contributed equally to this work. ${ }^{1}$ INSERM, CNRS, Institut de la Vision, 17 rue Moreau, Sorbonne Université, UPMC Univ Paris 06, F-75012 Paris, France

Full list of author information is available at the end of the article
}

capillaries, extravasation, and retinal edema. In this model, ECs subsequently proliferate and the edema resolves [1]. BRVO has also been shown to be associated with increased levels of chemokines and cytokines (including CCL2, which induces inflammatory monocytes (Mos) recruitment) in experimental BRVO $[1,2]$ in aqueous [3, 4] and in vitreous humor [5-7] of BRVO patients. In experimental BRVO, resident Iba1+ macrophages (rM $\phi s)$ are activated and blood-derived $\mathrm{M} \phi \mathrm{s}$ (iM $\phi s)$ are recruited [2] in the retina but their role in vascular remodeling in BRVO remains unknown. Mononuclear phagocytes (MPs) are a family of cells that

(c) The Author(s). 2019 Open Access This article is distributed under the terms of the Creative Commons Attribution 4.0 International License (http://creativecommons.org/licenses/by/4.0/), which permits unrestricted use, distribution, and 
include resident macrophages $(\mathrm{rM} \phi)$, such as microglial cells (MCs) and perivascular M $\phi$ s (vasM $\phi s)$ in the retina, and Mo-derived $\mathrm{M} \phi \mathrm{s}$ (iM $\phi \mathrm{s}$ ) that are recruited to the injured tissue upon chemokine induction. Blood monocytes can be divided into two subsets [8-10]: the CCR2 $2^{+}$"inflammatory" monocytes, the blood-born precursors of inflammatory macrophages and inflammatory dendritic cells, and the CCR2 ${ }^{-}$"non-inflammatory" monocytes that patrol the vasculature $[10,11]$ but also participate in the inflammatory reaction in certain affections, such as atherosclerosis [12]. Using the laser-induced BRVO model in mice, we here confirm that Moderived $M \varphi s$ are recruited to the occluded retina. Using Ccr2-deficient mice and clodronate-induced circulating Mos depletion, we identified two major subset of infiltrating MPs: 1/parenchymal $\mathrm{M} \phi \mathrm{s}$ (parM $\phi \mathrm{s}$ ) and 2/ vasM $\phi$ s that were recruited independently of CCL2/ CCR2 signaling. Mos depletion, but not CCR2 depletion, significantly exaggerated the wave of BRVO-induced EC apoptosis, suggesting that Mo-derived CCR2-negative vasM $\phi$ s protect $\mathrm{EC}$ in $\mathrm{BRVO}$ against apoptosis.

\section{Material and methods}

\section{Animals}

Eight to 12-weeks-old C57BL/6JRj male mice were purchased from Janvier SA (Le Genest-Saint-Isle, France). $\mathrm{Ccr} 2^{R F P / R F P}\left(\mathrm{~B} 6.129(\mathrm{Cg})-\mathrm{Ccr} 2^{\text {tm2.1Ifc }} / \mathrm{J}\right)$ mice were purchased from Jackson Laboratory (Bar Harbor, USA). Mice were maintained at the Institut de la Vision animal facility under pathogen-free conditions. All animals were housed in a 12-h/12-h light/dark cycle with food and water available ad libitum.

\section{Ethics statement}

All manipulations were performed in accordance with the association for research in vision and ophthalmology (ARVO) Statement for the Use of Animals in Ophthalmic and Vision Research. In addition, all the experimental procedures were permitted by the Institutional Animal Care and Use Committee, "Comité d'éthique pour l'expérimentation animale Charles Darwin" (ID Ce5/2012/013), which also specifically approved the study reported in the present manuscript.

\section{BRVO model}

Occlusion of one branch of the retinal vein was performed by laser photocoagulation as previously described [1, 13] with slight modifications. Pupils were dilated with tropicamide (Mydriaticum, Théa, France) and phenylephrin (Neosynephrine, Europhta, France). Animals were injected with $100 \mu \mathrm{l}$ of $1 \%$ fluorescein (FluoresceineSodique Faure 10\%, Novartis Pharma, France) for dye enhancement and were immediately anesthetized by inhalation of isoflurane (Axience,
France). Fundus was visualized with a slit lamp (BQ 900, Hagg-Streitt, Swiss) through a fundus laser lens (OFA $2.0 \mathrm{~mm}$, Ocular Instruments Inc., USA) positioned on the mouse cornea with eye gel (Lubrithal, DechraVetxx, France). Seven to 12 laser impacts $(0.5 \mathrm{~s}, 200 \mathrm{~mW}$, $50 \mu \mathrm{m}$ spot size, Laser Yag 532 Eyelite (Alcon, USA)) were performed on a superior vein, 2 to 3 disc diameter from the optic disc. The complete and the persistence of the occlusion were checked using fluorescent angiography with Micron III device (Phoenix Labs). Development of an eventual recanalization of the vein was checked during and at the end of the study by a control fundus.

Laser impacts were performed on retina away from any veins to exclude consequence of the laser alone. Mice in which retinal hemorrhage occurred or with incomplete occlusion were excluded from the study.

\section{RT-PCR}

At different times after BRVO, part of retina corresponding to the occluded area was carefully dissected in RNase-free conditions. Total RNA was isolated with Nucleospin RNAII (Macherey Nagel, France). Singlestranded cDNA was synthesized from total RNA (pretreated with DNaseI amplification grade) using oligo-dT as primer and superscript II reverse transcriptase (Life Technologies, France). Subsequent real-time polymerase chain reaction (RT-PCR) was performed using cDNA, Taqman, or SYBRgreen Gene Expression Master Mix (Life Technologies) and primers $(0.5 \mathrm{pmol} / \mu \mathrm{l})$ available upon request. Results were normalized by expression of S26. PCR reactions were performed in 45 cycles of $15 \mathrm{~s}$ at $95^{\circ} \mathrm{C}$ and $45 \mathrm{~s}$ at $60^{\circ} \mathrm{C}$. Primers used during the study are listed below.

\begin{tabular}{ll}
\hline S26_AS & AGCTCTGAATCGTGGTG \\
S26_S & AAGTTTGTCATTCGGAACATT \\
CCL2_AS & ACCCATTCCTTCTTGGGGTC \\
CCL2_S & GATGCAGTTAACGCCCCACT \\
CCR2_AS & GGCATGAGGCTGTCAG \\
CCR2_S & ATTGTCCATGTTGTCATAGAT \\
ICAM1_AS & CAGGGTGAGGTCCTTGCCTA \\
ICAM1_S & TCCGCTACCATCACCGTGTA \\
F4/80_AS & TGGAAGTGGATGGCATAGATG \\
F4/80_S & TTCACTGTCTGCTCAACG \\
CD11b_AS & ATGCTGTGCTGTCCTCTCTG \\
CD11b_S & ATCTCTGCTGGCTTCCAGT \\
\hline
\end{tabular}

\section{Immunohistochemistry}

Mice were killed by $\mathrm{CO}_{2}$ inhalation, and the eyes were enucleated and fixed in $4 \%$ paraformaldehyde for $30 \mathrm{~min}$ at room temperature. After several washes in PBS, the 
cornea and lens were removed and the retina was carefully detached from RPE/choroid/sclera. The retinas were incubated overnight with primary antibodies (goat anti-collagen IV, 1:100, Serotec, USA; rabbit anti-Iba1, 1: 400, 1:500, Millipore, USA) in PBS supplemented with 0.5\% Triton X-100, followed by incubation with appropriate Alexa-coupled secondary antibodies (Life Technologies). The retinas were flatmounted and viewed with a fluorescence microscope (DM5500B, Leica, France) or with a confocal microscope (FV1000, Olympus, France).

All cell counting was performed in the inner retina (GCL/IPL). Vessel lengths and vascular areas were calculated with the "angiogenesis tube formation" add-in [14] available on MetaMorph 2D software (Molecular Devices, France).

\section{MP tracing by systemic EdU injection and Mo depletion with clodronate liposomes}

Repeated systemic EdU injections are able to stain monocytes in bone marrow as previously described [15]. With this procedure, $76 \%$ of CD115+ circulating monocytes showed EdU incorporation (during 4days). Briefly, mice were treated daily by three intraperitoneal injections of EdU (50 mg/kg, Life Technologies) 1 day before BRVO and in the three following days [1]. Intraretinal proliferation was measured traced by intravitreous injections of EdU $(6.7 \mu \mathrm{g} /$ $\mu \mathrm{l}, 2 \mu \mathrm{l} / \mathrm{eye})$. Additionally, mice were intravenously injected with $100 \mu \mathrm{l}$ of control liposomes (placebo control liposomes for clophosome-A) or liposomes containing clodronate (clophosome-A-anionic liposomal clodronate for macrophage depletion, FormuMax) the day of occlusion and the two following days leading to a decrease of circulation monocyte population of $84 \%$ [15]. The eyes were removed, and retinal flatmounts were labeled with Iba1 and revealed for EdU incorporation (Click-iT EdU Imaging Kit, Life Technologies) for the quantification of Iba1+ and EdU+ cells.

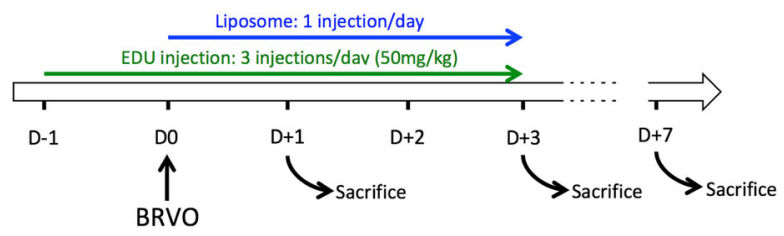

\section{Terminal deoxynucleotidyl transferased UTP nick end labeling}

After immunohistochemistry, retinal flatmounts were pretreated with frozen methanol for $20 \mathrm{~min}$ and then frozen methanol/acetic acid (2:1) for another $20 \mathrm{~min}$. After washing with PBS, flatmounts were incubated overnight at $4{ }^{\circ} \mathrm{C}$ with the reaction mixture as described by the manufacturer's protocol (In Situ Cell Death
Detection Kit, Roche Diagnostics, USA) and then for 90 min at $37^{\circ} \mathrm{C}$. After reaction was stopped by washing with PBS at RT, nuclei were counterstained with Hoechst (Sigma-Aldrich, France). The retinas were mounted, viewed, and photographed with a fluorescence microscope (DM5500B, Leica) or with a confocal microscope (FV1000, Olympus).

\section{Statistical analysis}

GraphPad Prism 5 (GraphPad Software, San Diego, USA) was used for data analysis and graphic representation. All values are reported as mean \pm SEM. Statistical analysis is described in the legend of each figure. $P<0.05$ was considered statistically significant.

\section{Results}

BRVO increases the number of perivascular and parenchymal mononuclear phagocytes

To better characterize the inflammatory processes in BRVO, we first quantified by RT-PCR the mRNA expression of intercellular adhesion molecule 1 (Icam-1), involved in leukocyte diapedesis, and the mononuclear phagocyte (MP) markers $C d 11 b$ and $F 4 / 80$ at day 1 , day 3 , and day 7 . The upstream retinas of the occluded veins are easily recognizable by the dilated vein and the diffused retinal extravasations. These areas were carefully dissected in RNase-free conditions (Fig. 1a). All three transcripts were strongly and significantly induced at all investigated time points compared to non-occluded (white bar-day 0), peaking at day 3 (Fig. 1b). Analysis at day 3 and day 7 of Iba1 (green staining) and collagen IV (ColIV, red staining) double-labeled retinal flatmounts on the occluded area (rectangle-Fig. 1a) revealed two distinct populations of MPs in the inner retina, parenchymal and perivascular MPs. Vascular Mфs were distinguished from parenchymal M $\phi$ s by their more rounded and elongated cell bodies (Fig. 2b inset) and their physical contact with the ColIV+ retinal vein. The density of parenchymal macrophages (parM $\phi$ s), located in the ganglion cell layer with no direct contact to the vasculature, increased by $46.9 \%$ at day $3\left(190.1 \pm 16.3\right.$ cells $\left./ \mathrm{mm}^{2}\right)$ and $+68.3 \%$ at day $7\left(217.8 \pm 36.7\right.$ cells $\left./ \mathrm{mm}^{2}\right)$ compared to non-occluded retina $\left(129.4 \pm 24.0\right.$ cells $/ \mathrm{mm}^{2}$; Fig. $\left.1 \mathrm{c}-\mathrm{e}\right)$. Similarly, the number of elongated, ramified perivascular macrophages (vasM $\phi$ s) located around the occluded vein appeared more numerous compared to non-occluded retina (Fig. 1f, g) [16]. Cell counts of vasM $\phi$ s per vein length at day $3(24.22 \pm 3.8$ cells $/ \mathrm{mm})$ and at day 7 $(25.37 \pm 3.5$ cells $/ \mathrm{mm})$ revealed a nearly twofold significant increase of vasM $\phi$ s compared to non-occluded retina $(12.81 \pm 2.1$ cells $/ \mathrm{mm})$ at both time points (Fig. 1h).

Together our analysis shows that the number of both, vasM $\phi s$ and parM $\phi s$, significantly increase after BRVO at 3 days and for the whole of the observation period. 

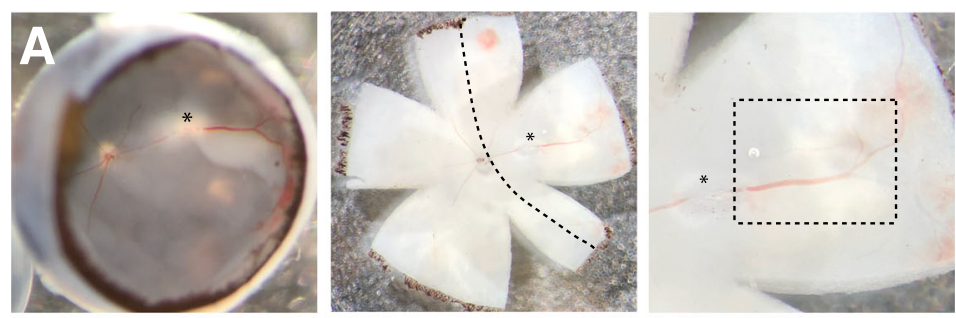

B
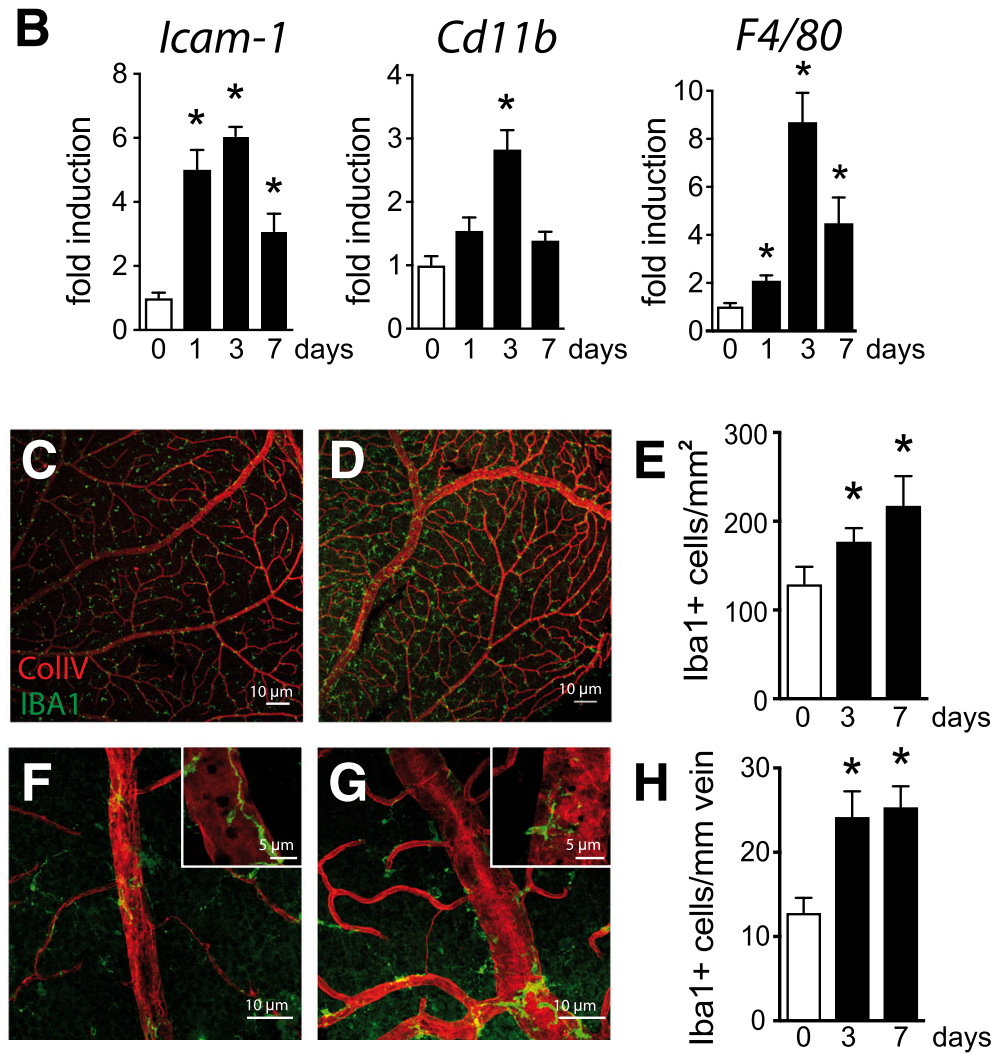

Fig. 1 BRVO increases the number of perivascular and parenchymal mononuclear phagocytes. Photographs of the eyecup and retinal flatmount of the retina with the visible vein occlusion site $\left(^{*}\right)$, dilated vein, and hemorrhages at day 1 after BRVO. Dotted line shows the dissected area of the retina used for RT-PCR and the dotted rectangle the area where perivascular M $\varphi$ s were counted during the study (a). Icam-1, cd11b, and F4/ 80 real-time RT-PCRs in the occluded retina at indicated time points. The results were normalized to S26 expression. Values in histograms are mean \pm SEM of mRNA expression of occluded area from 4 eyes per group $\left(\mathbf{b}, n=6\right.$, Mann-Whitney $t$ test; Icam-1: ${ }^{*} P=0.0095$ day 0 versus day 1 , ${ }^{*} P=0.0047$ day 0 versus day 3 , and ${ }^{*} P=0.0095$ day 0 versus day $7 ; C d 11 b:{ }^{*} P=0.0095$ day 0 versus day $3 ; F 4 / 80:{ }^{*} P=0.0424$ day 0 versus day 1 , ${ }^{*} P=0.0047$ day 0 versus day 3 , and ${ }^{*} P=0.0095$ day 0 versus day 7). Representative images of the inner retina of Iba1 (green) and ColIV (red) double-labeled retinal flatmounts of control (c) and occluded vein area 3 days after occlusion (d). Magnification on control vein in the upstream retina $(\mathbf{f})$ and on the occluded vein $(\mathbf{g}) 3$ days after occlusion. Quantification of the parenchymal Iba $1^{+} \mathrm{M} \varphi s$ per square millimeter (e, MannWhitney $t$ test, $\mid \mathrm{baa} 1^{+}$cells $/ \mathrm{mm}$ of vein: day 0 versus d3day ${ }^{*} P=0.0229$ and day 0 versus day $7{ }^{*} P=0.0424$ ) and the number of perivascular $\mid \mathrm{Iba} 1^{+}$ M $\varphi s$ per millimeter of vein $\left(\mathbf{h}\right.$, Mann-Whitney $t$ test, $\mid \mathrm{lba} 1^{+}$cells $/ \mathrm{mm}^{2}$ : day 0 versus day $3{ }^{*} P=0.0229$ and day 0 versus day $\left.7{ }^{*} P=0.0424\right)$ of control retinas and in the occluded vein 3 and 7 days after the occlusion. Icam-1, intracellular adhesion molecule 1; Iba1, ionized calcium-binding adapter molecule 1. Scale bar $\mathbf{c}$ and $\mathbf{d}=10 \mu \mathrm{m}$. Inset $=5 \mu \mathrm{m}$. Scale bar $\mathbf{e}$ and $\mathbf{f}=50 \mu \mathrm{m}$

The increased population of lba-1+ cells in the inner retina after BRVO is partly due to blood-derived macrophage infiltration

To evaluate the participation of monocyte recruitment from the bloodstream versus resident $M \phi s$ and $M C s$ in BRVO-induced MP accumulation at day 3, we injected mice three times a day for 4 days (from $24 \mathrm{~h}$ prior to occlusion to day 3) intraperitoneally with the traceable nucleotide EdU, which we showed marks $75 \%$ of circulating $\mathrm{CD}_{115^{+}}$monocytes, but not resident retinal MCs and M $\phi s$ [15]. Furthermore, we inhibited the accumulation of Mo-derived M $\phi$ s by additional daily intravenous (day 0 to day 2) of either empty control liposomes or clodronate-containing liposomes, which we showed to 

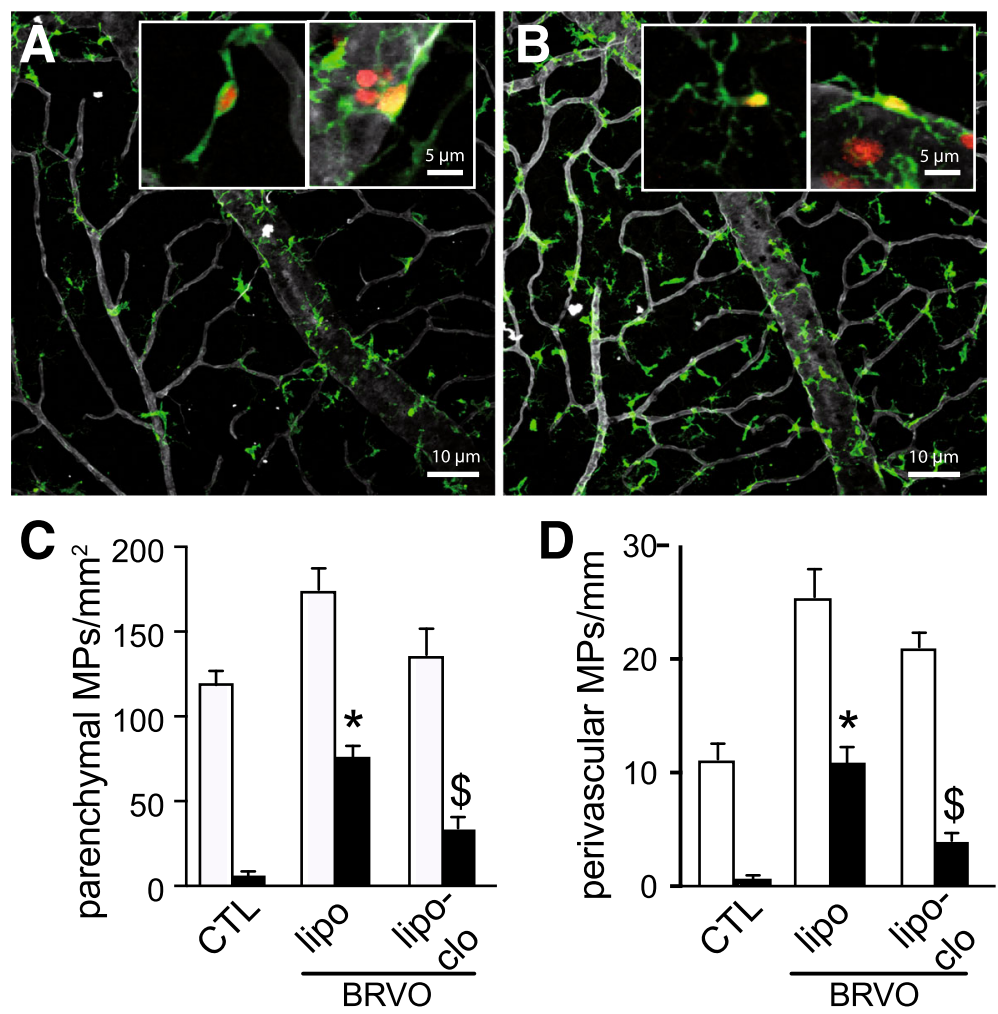

Iba1+ cells

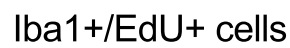

Fig. 2 The increased population of Iba-1+ cells in the inner retina after BRVO is partly due to blood-derived macrophage infiltration. Representative images of the inner aspect of Ibal (green), ColIV (white), and EdU (red) triple-labeled retina of control animals (a) and 3 days after BRVO (b) that were intraperitoneally injected with EdU for 4 days. The insets depict representative EdU+lba1+ parenchymal (left panel) and

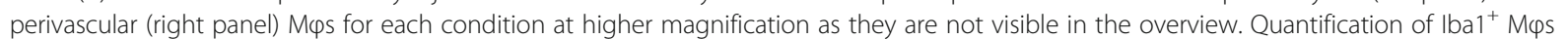

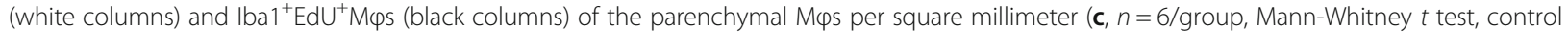
parenchymal Iba ${ }^{+} \mathrm{EdU}{ }^{+} \mathrm{M} \varphi$ s versus BRVO liposome parenchymal Iba $1^{+} \mathrm{EdU}{ }^{+} \mathrm{M} \varphi{ }^{*}{ }^{*}=0.0143$; BRVO liposome parenchymal Iba $1^{+} \mathrm{EdU}{ }^{+} \mathrm{M} \varphi s$ versus

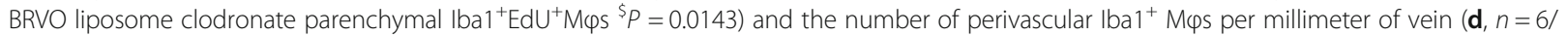
group, Mann-Whitney $t$ test, control perivascular Iba ${ }^{+} E d U^{+} M \varphi s$ versus BRVO liposome parenchymal Iba $1^{+} E d U^{+} M \varphi s{ }^{*} P=0.0143$; BRVO liposome parenchymal Iba $1^{+} E d U^{+} M \varphi s$ versus BRVO liposome clodronate perivascular $\left.\mid \mathrm{bal} 1^{+} \mathrm{EdU}{ }^{+} \mathrm{M} \varphi \mathrm{s}^{\$} P=0.0095\right)$ of control retinas and retinas from BRVO mice receiving intravenous control liposome injections (lipo) or clodronate liposome injections (lipo-clo). BRVO, branch retinal vein occlusion; lipo, control liposome-treated; lipo-clo, liposome clodronate-treated; Iba1, ionized calcium-binding adapter molecule 1. Scale bar a and $\mathbf{b}=10 \mu \mathrm{m}$. Inset $=5 \mu \mathrm{m}$

lower the number of circulating monocytes by at least $85 \%$ for $24 \mathrm{~h}$ [15].

Micrographs of retinal areas of the mid-periphery of EdU (red staining), Iba-1 (green staining), and ColIV (white staining) triple-stained retinal flatmounts of non-occluded eyes from mice that were repeatedly injected with EdU for 4 days without liposome injection (CTL-Fig. 2a) and of the occluded vein of 3 days post-BRVO mice (BRVO-Fig. 2b) confirm the accumulation of $\mathrm{Iba}^{+}{ }^{+} \mathrm{parM} \phi \mathrm{s}$ and vasM $\phi \mathrm{s}$ upstream of the vein occlusion and surrounding retina described above. $\mathrm{EdU}^{+} \mathrm{Iba1}^{+}$parM $\phi$ s and vasM $\phi$ s (inset Fig. 2a, b) were visible in both occluded and non-occluded retina. However, quantification of $\mathrm{EdU}^{+} \mathrm{Iba}^{+}$ and $\mathrm{EdU}^{-} \mathrm{Iba1}^{+}$parM $\phi$ s (Fig. 2c) and vasM $\phi$ s (Fig. 2d) revealed that $\mathrm{EdU}^{+} \mathrm{Iba1}^{+} \mathrm{M} \phi \mathrm{s}$ are rare in the non- occluded control eyes (CTL). Control liposomeinjected experimental BRVO mice revealed that around half of the $\mathrm{Iba1}^{+}$parM $\phi$ s and vasM $\phi$ s were EdU positive. This could be due either to the recruitment of $\mathrm{EdU}^{+} \mathrm{Mo}$ that differentiate into $\mathrm{EdU}^{+} \mathrm{Iba} 1^{+} \mathrm{M} \phi \mathrm{s}$ or to EdU incorporation into locally proliferating M $\phi s$. To differentiate the two, we depleted the mice of their circulating Mos by intravenous liposome clodronate injections, which very significantly reduced the numbers of $\mathrm{EdU}^{+} \mathrm{Iba} 1^{+}$ parM $\phi s$ and vasM $\phi s$ in both populations. The $I b a 1^{+} E-$ $\mathrm{dU}^{\text {neg }}$ cells (white bars minus the black bars) stayed stable suggesting that they were not affected by the intravenous injection of clodronate liposomes.

In conclusion, our data demonstrates that $\operatorname{parM} \phi s$ and vasM $\phi$ s that accumulate after BRVO are at least in part 
derived from Mo and that the accumulation of Mo-derived parM $\phi$ s and vasM $\phi s$ can efficiently be inhibited by Mo depletion in experimental BRVO.

\section{CCR2 ${ }^{+}$Mo-derived $M \varphi$ participates in parM $\varphi$ s but not in vas $M \varphi s$ accumulation after vein occlusion}

CCL2 strongly participates in the recruitment of $\mathrm{CCR}^{+}$monocytes, which differentiate in the tissue into inflammatory $\mathrm{M} \phi \mathrm{s}$ during the early phases of the inflammatory response [8-10]. Quantification of mRNA by RT-PCR revealed a strong induction of $\mathrm{Ccl} 2$ and $\mathrm{Ccr} 2$ at day 1 and day 3 before falling back to basal expression levels at 7 days following the vein occlusion. To investigate the participation of CCR2 ${ }^{+}$ Mos in the accumulation of Mo-derived parM $\phi s$ and vasM $\phi$ s, we submitted wildtype and $C c r 2^{R F P / R F P}$ mice to $\mathrm{BRVO}$ and quantified M $\phi \mathrm{s}$ on Iba1 (green staining) and CollIV (red staining) double-labeled retinal flatmounts. We previously demonstrated that red fluorescent protein (RFP) expression cannot be used as a marker to determine whether a $M \phi$ is derived from a $\mathrm{CCR}^{+}$monocyte as the $\mathrm{Ccr} 2$ promotor activity that drives the RFP expression in $C c r^{R F P / R F P}$ mice quickly comes to a halt after tissue infiltration [15]. The BRVO-induced increase in a number of parM $\phi s$ (Fig. 3b, c) observed in wildtype mice was
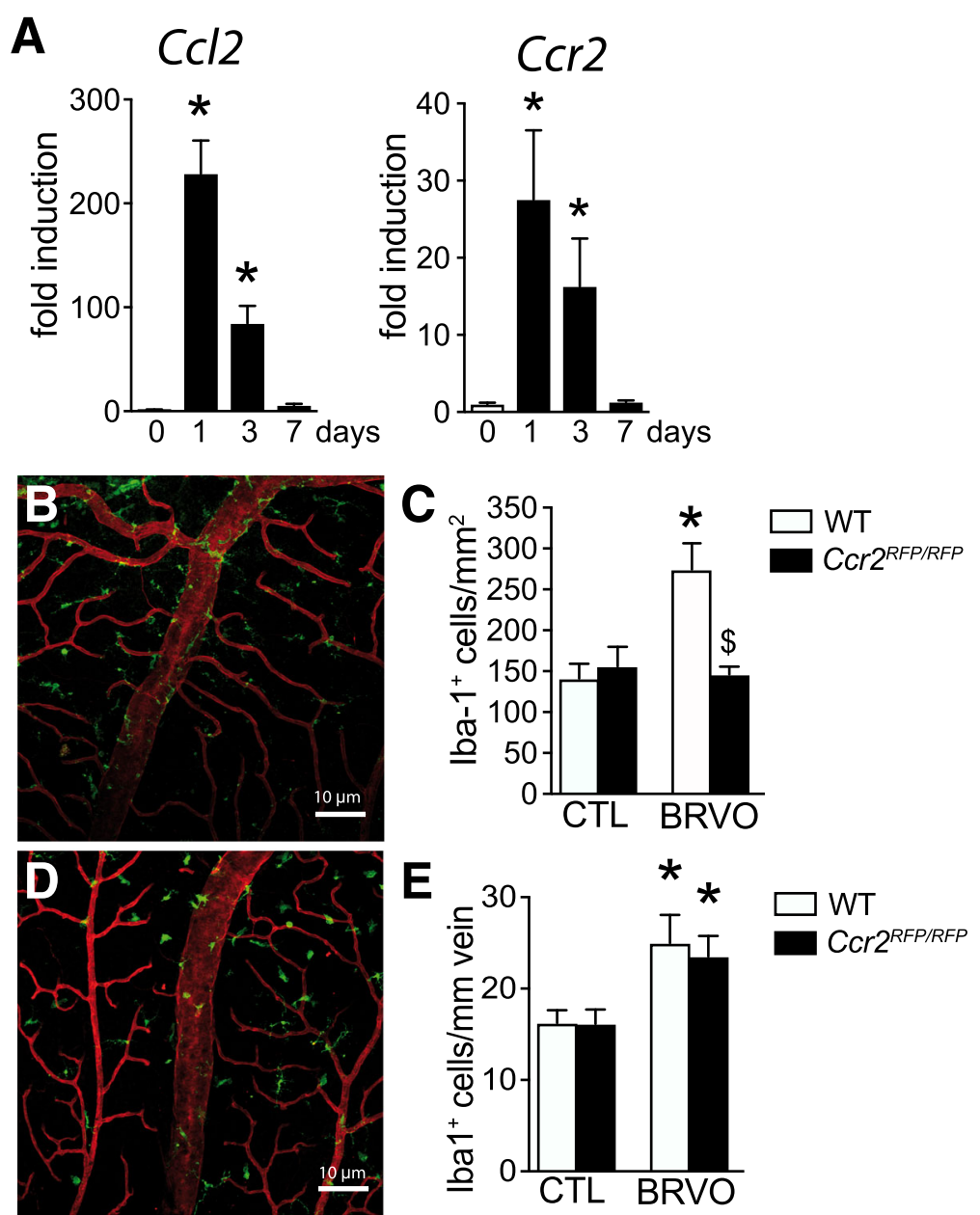

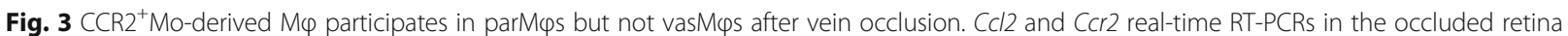
at indicated time points (a). The results were normalized to S26 expression. Values in histograms are the mean \pm SEM of mRNA expression on the occluded area from 4 eyes per group (a; $n=4 /$ group, Mann-Whitney $t$ test, $\mathrm{Ccl} 2$ and $\mathrm{Ccr} 2$ expression at day 0 versus day 1 and versus day 3 , ${ }^{*} P=0.0286$ ). Representative images of the inner retina of Ibal (green) and ColIV (red) double-labeled retinal flatmounts of occluded vein in WT (b) and in $\mathrm{CCr} 2^{\text {GFP/GFP }}$ (d) mice 3 days after vein occlusion. Quantification of the parenchymal Iba1 ${ }^{+} \mathrm{M} \varphi$ s per square millimeter $(\mathbf{c} ; n=4 \mathrm{WT}$ and $n=6$ C cr2 ${ }^{\text {GFP/GFP }}$, Mann-Whitney $t$ test, $\mid \mathrm{bba} 1^{+}$cells $/ \mathrm{mm}^{2}$ in control versus BRVO ${ }^{*} P=0.0143$; BRVO in WT versus in $C \mathrm{cr} 2^{G F P / G F P}$ mice $\left.{ }^{\$} P=0.0246\right)$ and the number of perivascular $\mid \mathrm{ba} 1^{+} \mathrm{M} \varphi s$ per millimeter of vein $\left(\mathbf{e}, \mathrm{iba} 1^{+}\right.$cells $/ \mathrm{mm}$ vein in control versus BRVO for $\mathrm{WT},{ }^{*} P=0,0143$, and $\mathrm{CCr}{ }^{G F P / G F P}$ mice, $\left.{ }^{*} P=0.0095\right)$ of control retinas and in the occluded vein 3 days after the occlusion. Ccl2, chemokine ligand 2; Ccr2, C-C chemokine receptor type 2; BRVO, branch retinal vein occlusion; Iba1, ionized calcium-binding adapter molecule 1. Scale bar $\mathbf{b}$ and $\mathbf{d}=10 \mu \mathrm{m}$. Inset $=5 \mu \mathrm{m}$ 
significantly reduced in $C c r 2^{R F P / R F P}$ mice presenting the same level of parM $\phi$ s than in non-occluded retina (CTL). Interestingly, Ccr2 deficiency had no effect on the accumulation of vasM $\phi$ s (Fig. 3d, e) after BRVO.

These results suggested that contrary to the increase of $\operatorname{parM} \phi s$, increased numbers of vas $M \phi s$ are not derived from $\mathrm{CCR}^{+}$monocytes.

\section{Mo-derived perivascular M $\varphi s$ inhibit endothelial cell apoptosis}

We have previously showed that ECs of the occluded vein undergo a wave of TUNEL+ apoptosis during the first 3 days following the obstruction followed by EC proliferation that mainly occurs thereafter [1]. TUNEL (green staining) and ColIV (red staining) double labeling of retinal flatmounts 3 days after experimental vein occlusion revealed $\mathrm{TUNEL}^{+} \mathrm{ColIV}^{+}$apoptotic ECs on occluded veins of control liposome-treated mice (Fig. 4a) as previously described for untreated animals [1]. However, TUNEL ${ }^{+} \mathrm{ColIV}^{+}$apoptotic ECs appeared more numerous in liposome clodronate-treated mice (Fig. 4b), which lack the Mo-derived vasM $\phi$ s accumulation (Fig. 2). Interestingly, quantification of the number of TUNEL ${ }^{+-}$ ColIV $^{+}$apoptotic ECs upstream of the vein occlusion was significantly increased in liposome clodronatetreated mice (Fig. 4c) but showed no difference between the wildtype and $C \mathrm{cr} 2^{R F P / R F P}$ mice (Fig. $4 \mathrm{~d}$ ).

Taken together, as others and we previously showed that intravenous liposome clodronate $[17,18]$ does not directly induce EC apoptosis, our data suggests that the recruitment of CCR $2^{\text {neg }} \mathrm{Mo}$-derived vasM $\phi$ s protects $\mathrm{EC}$ of the occluded vein from apoptosis.

\section{Discussion}

We have previously shown that experimental BRVO is associated with an acute wave of endothelial cell (EC) apoptosis of the occluded vein and the upstream capillaries followed by the subsequent proliferation of the ECs [1]. We here show that experimental BRVO is accompanied by an increased expression of Icam-1 (that plays a central role in leukocyte recruitment to an injured tissue [19]), CD11b (an integrin that is more strongly expressed in Mo than MCs; Immgen.org), and F4/80 (EMR1, strongly expressed in macrophages during inflammation). Indeed, we demonstrate that $\mathrm{Iba} 1^{+} \mathrm{M} \phi \mathrm{s}$ accumulate after BRVO and we identified two subtypes of Mo-derived M $\phi s$ : parM $\phi s$, accumulating in the inner retinal layer upstream of the vein occlusion, and more elongated vasM $\phi s$, which are located in and around the vessel wall of the occluded vein. Our experiments demonstrated a significant accumulation as soon as post-occlusion day 3 that remained elevated through day 7 . The comparatively lower CD11b and F4/80 expression by RT-PCR at day 7 compared to day 3 in the occluded retina is therefore not the result of a decrease of MP numbers but likely the result of differences in the level of expression during $M \phi$ differentiation and activation. Using a combination of traceable nucleotide EdU injections and liposome clodronate-induced depletion of circulating, we show that the accumulation of both $\operatorname{parM} \varphi s$ and vasM $\phi s$ is to a considerable extent due to the recruitment and infiltration of Mo-derived M $\phi$ s. Intravenous liposome clodronate injection decreases iba $1^{+} E_{d U^{+}}$cell density counted 3 days after BRVO without affecting the ibal ${ }^{+} E d U^{\text {neg }}$ cell population. We controlled that intravenous injection of liposome clodronate
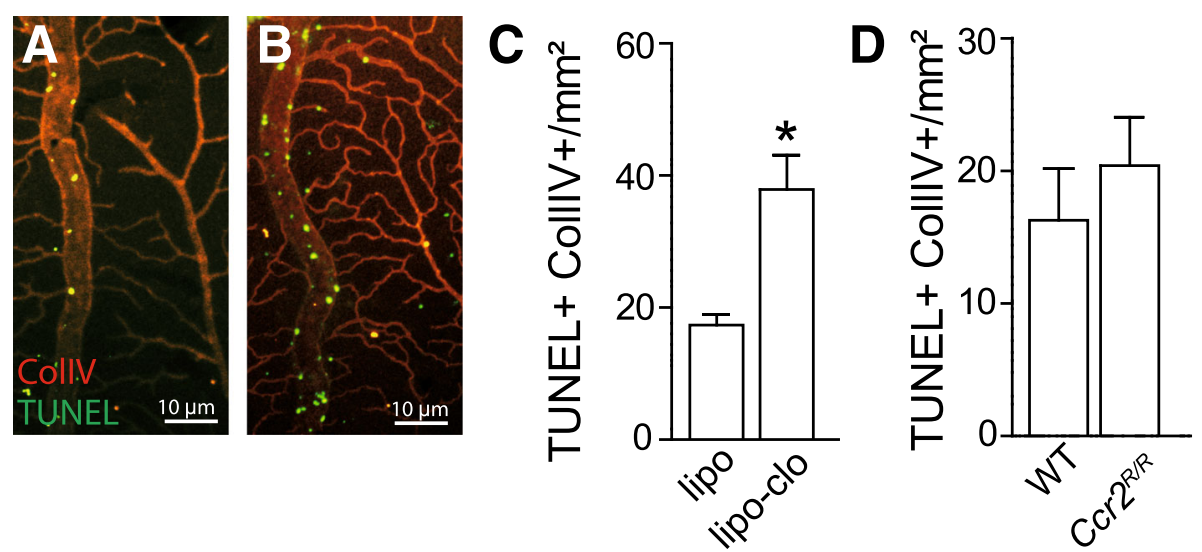

Fig. 4 Mo-derived perivascular M $\varphi$ s inhibit endothelial cell apoptosis. Representative images of the inner aspect of the of CoIIV (red) and TUNEL (green) double staining of the occluded vein area 3 days after the occlusion in control liposome (a) and clodronate liposome (b)-treated mice. Quantification of ColIV+TUNEL+ cells on the occluded area and comparison between control liposome and clodronate liposome-injected mice (c, $n=8$, Mann-Whitney $t$ test, $\left.{ }^{*} P=0.0004\right)$ and between WT and $C c r 2^{G F P / G F P}$ mice (d). lipo, control liposome-treated; lipo-clo, liposome clodronatetreated; ColIV, collagen IV; TUNEL, terminal deoxynucleotidyl tranferase dUTP nick end labeling. Scale bar $\mathbf{a}$ and $\mathbf{b}=10 \mu \mathrm{m}$ 
does not modify the density of both vasM $\phi$ s and parM $\phi s$ in non-occluded animals (data not shown).

We observed a significant transient increase of Ccr2 mRNA, a cytokine receptor that is strongly expressed on classical CCR2+Mo but is quickly downregulated upon recruitment to the tissue [15] and Ccl2 mRNA, its predominant cytokine. Using wildtype and $C C R 2^{R F P / R F P}$ mice, we demonstrate that CCR2+Mo recruitment does indeed participate in the $\mathrm{M} \phi$ infiltration after BRVO. Surprisingly, Ccr2 deficiency very strongly blunted the accumulation of parM $\phi$ s but had no effect on vasM $\phi$ s.

The observation that vasM $\phi s$ can be strongly inhibited by Mo depletion, but not by Ccr2 ablation, strongly suggests that the vasM $\phi$ s were recruited through a CCR2independent mechanism. Non-classical monocytes that do not express Ccr2 and that are involved in other pathologies $[11,20]$ would seem a likely candidate. Indeed, a subgroup of non-classical Mo expressing high levels of the chemokine receptor CXCR4 has previously been shown to be angio-protective [21,22] as it produces high level of VEGF [20] and specifically accumulates in the perivascular area in diseases such as autoimmune encephalomyelitis (EAE) [23].

Similarly, our analysis of endothelial cell apoptosis 3 days post-BRVO in control liposome and liposome clodronate-treated mice, and wildtype and $C c r 2^{R F P / R F P}$ mice clearly shows that the inhibition of vasM $\phi$ s recruitment, but not parM $\phi s$, leads to a dramatic increase of EC apoptosis in the occluded vein.

\section{Conclusion}

Taken together, our study demonstrates for the first time an important role of blood-derived vasM $\phi$ s in the vessel remodeling after BRVO. Our findings show a vasoprotective effect of blood-derived vasM $\phi$ s that should be taken into consideration in the development of future therapies.

\section{Abbreviations \\ BRVO: Branch retinal vein occlusion; Ccl2: C-C chemokine ligand 2; Ccr2: C-C chemokine receptor type 2; ColIV: Collagen IV; EC: Endothelial cell; EdU: 5- Ethynyl-2'-deoxyuridine; Iba1: Ionized calcium-binding adapter molecule 1; Icam-1: Intracellular adhesion molecule 1; iM $\varphi$ s: Infiltrated macrophages; lipo: Control liposome-treated; lipo-clo: Liposome clodronate-treated;

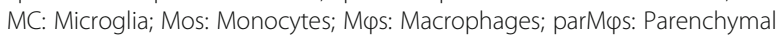 macrophages; rM $\varphi$ : Resident macrophages; TUNEL: Terminal deoxynucleotidyl tranferase dUTP nick end labeling; vasM $\varphi$ s: Perivascular macrophages}

\section{Acknowledgements}

We wish to thank J. Degardin, M. Simonetti, and Q. Cesar from the animal facility of the Institut de la Vision.

\section{Authors' contributions}

CR made substantial contributions to the data analysis and interpretation. CR drafted the manuscript, gave the final approval of the version to be published, and agreed to be accountable for all aspects of the work. ED made an important contribution in the data acquisition and analysis. WR, $X G$, MP, JAS, and FS made substantial contributions to the analysis and interpretation of data, revised the manuscript critically for important intellectual content, gave final approval of the version to be published, and agreed to be accountable for all aspects of the work. FS made substantial contributions to the study conception and design and the analysis and interpretation of data, drafted the manuscript, gave the final approval of the version to be published, and agreed to be accountable for all aspects of the work. All authors read and approved the final manuscript.

\section{Funding}

This work was supported by the grants from INSERM and LABEX LIFESENSES (ANR-10-LABEX-65).

\section{Availability of data and materials}

All data generated or analyzed during this study are included in this published article. The datasets used and/or analyzed during the current study are also available from the corresponding author on reasonable request.

\section{Ethics approval and consent to participate}

All manipulations were performed in accordance with the association for research in vision and ophthalmology (ARVO) Statement for the Use of Animals in Ophthalmic and Vision Research. In addition, all the experimental procedures were permitted by the Institutional Animal Care and Use Committee, "Comité d'éthique pour l'expérimentation animale Charles Darwin" (ID Ce5/2012/013), which also specifically approved the study reported in the present manuscript.

\section{Consent for publication}

Not applicable.

\section{Competing interests}

The authors declare that they have no competing interests.

\section{Author details}

${ }^{1}$ INSERM, CNRS, Institut de la Vision, 17 rue Moreau, Sorbonne Université, UPMC Univ Paris 06, F-75012 Paris, France. ${ }^{2}$ Université François Rabelais de Tours, CNRS, GICC UMR 7292, Tours, France. ${ }^{3}$ Centre Hospitalier National d'Ophtalmologie des Quinze-Vingts, INSERM-DHOS CIC 1423, F-75012 Paris, France. ${ }^{4}$ Berlin Institute of Health, Berlin, Germany.

Received: 8 March 2019 Accepted: 16 July 2019

Published online: 27 July 2019

\section{References}

1. Dominguez E, Raoul W, Calippe B, Sahel J-A, Guillonneau X, Paques M, et al. Experimental branch retinal vein occlusion induces upstream pericyte loss and vascular destabilization. PLoS One. 2015;10(7):e0132644.

2. Ebneter A, Kokona D, Schneider N, Zinkernagel MS. Microglia activation and recruitment of circulating macrophages during ischemic experimental branch retinal vein occlusion. Investig Ophthalmol Vis Sci. 2017;58:944-53.

3. Feng J, Zhao T, Zhang Y, Ma Y, Jiang Y. Differences in aqueous concentrations of cytokines in macular edema secondary to branch and central retinal vein occlusion. PLoS One. 2013;8(7):e68149.

4. Kaneda S, Miyazaki D, Sasaki S, Yakura K, Terasaka Y, Miyake K, et al. Multivariate analyses of inflammatory cytokines in eyes with branch retinal vein occlusion: relationships to bevacizumab treatment. Invest Ophthalmol Vis Sci. 2011;52:2982-8.

5. Fonollosa A, Garcia-Arumi J, Santos E, Macia C, Fernandez P, Segura R, et al. Vitreous levels of interleukine-8 and monocyte chemoattractant protein-1 in macular oedema with branch retinal vein occlusion. Eye. 2010;24:1284-90.

6. Yoshimura T, Sonoda KH, Sugahara M, Mochizuki Y, Enaida H, Oshima Y, et al. Comprehensive analysis of inflammatory immune mediators in vitreoretinal diseases. PLoS One. 2009;4(12):e8158.

7. Pfister M, Rothweiler F, Michaelis M, Cinatl J, Schubert R, Koch FH, et al. Correlation of inflammatory and proangiogenic cytokines from undiluted vitreous samples with spectral domain OCT scans, in untreated branch retinal vein occlusion. Clin Ophthalmol. 2013;7:1061-7.

8. Geissmann F, Manz MG, Jung S, Sieweke MH, Ley K. Development of monocytes, macrophages and dendritic cells. Science (80- ). 2010;327:656-61. 
9. Swirski FK, Libby P, Aikawa E, Alcaide P, Luscinskas FW, Weissleder R, et al. Ly6Chi monocytes dominate hypercholesterolemia-associated monocytosis and give rise to macrophages in atheromata. J Clin Invest. 2007;117:195-205.

10. Tsou CL, Peters W, Si Y, Slaymaker S, Aslanian AM, Weisberg SP, et al. Critical roles for CCR2 and MCP-3 in monocyte mobilization from bone marrow and recruitment to inflammatory sites. J Clin Invest. 2007;1 17:902-9.

11. Geissmann F, Jung S, Littman DR. Blood monocytes consist of two principal subsets with distinct migratory properties. Immunity. 2003;19:71-82.

12. Combadière C, Potteaux S, Gao JL, Esposito B, Casanova S, Lee EJ, et al. Decreased atherosclerotic lesion formation in CX3CR1/apolipoprotein E double knockout mice. Circulation. 2003;107:1009-16.

13. Ebneter A, Agca C, Dysli C, Zinkernagel MS. Investigation of retinal morphology alterations using spectral domain optical coherence tomography in a mouse model of retinal branch and central retinal vein occlusion. PLoS One. 2015:10:1-15.

14. Zudaire E, Gambardella L, Kurcz C, Vermeren S. A computational tool for quantitative analysis of vascular networks. PLoS One. 2011;6:1-12.

15. Sennlaub F, Auvynet C, Calippe B, Lavalette S, Poupel L, Hu SJ, et al. CCR2+ monocytes infiltrate atrophic lesions in age-related macular disease and mediate photoreceptor degeneration in experimental subretinal inflammation in Cx3cr1 deficient mice. EMBO Mol Med. 2013;5:1775-93.

16. $\mathrm{X} u \mathrm{H}$, Chen M, Mayer EJ, Forrester J V., Dick AD. Turnover of resident retinal microglia in the normal adult mouse. Glia. 2007;55(11):1189-98.

17. Danenberg HD, Fishbein I, Gao J, Reich R, Gati I, Moerman E, et al. Macrophage depletion by clodronate-containing liposomes reduces neointimal formation after balloon injury in rats. Circulation. 2002;106(5): 599-605.

18. Checchin D, Sennlaub F, Levavasseur E, Leduc M. Potential role of microglia in retinal blood vessel formation. Invest Ophthalmol Vis Sci. 2006;47(8): 3595-602.

19. Nourshargh S, Alon R. Leukocyte migration into inflamed tissues. Immunity. 2014;41:694-707.

20. Nahrendorf M, Swirski FK, Aikawa E, Stangenberg L, Wurdinger T, Figueiredo $J-L$, et al. The healing myocardium sequentially mobilizes two monocyte subsets with divergent and complementary functions. J Exp Med. 2007: 204(12):3037-47.

21. Shantsila E, Tapp LD, Wrigley BJ, Montoro-Garcia S, Lip GYH. CXCR4 positive and angiogenic monocytes in myocardial infarction. Thromb Haemost. 2013;109(2):255-62.

22. Liehn EA, Tuchscheerer N, Kanzler I, Drechsler M, Fraemohs L, Schuh A, et al. Double-edged role of the CXCL12/CXCR4 axis in experimental myocardial infarction. J Am Coll Cardiol. 2011;58(23):2415-23.

23. McCandless EE, Wang Q, Woerner BM, Harper JM, Klein RS. CXCL12 limits inflammation by localizing mononuclear. J Immunol. 2006;1:8053-64.

\section{Publisher's Note}

Springer Nature remains neutral with regard to jurisdictional claims in published maps and institutional affiliations.

Ready to submit your research? Choose BMC and benefit from:

- fast, convenient online submission

- thorough peer review by experienced researchers in your field

- rapid publication on acceptance

- support for research data, including large and complex data types

- gold Open Access which fosters wider collaboration and increased citations

- maximum visibility for your research: over $100 \mathrm{M}$ website views per year

At $\mathrm{BMC}$, research is always in progress.

Learn more biomedcentral.com/submissions 